\title{
Characterization of Internal Organ Motion Using Skin Marker Positions
}

\author{
Ali Khamene ${ }^{1}$, Jan K. Warzelhan ${ }^{1}$, Sebastian Vogt ${ }^{1}$, Daniel Elgort ${ }^{2}$, \\ Christophe Chefd'Hotel ${ }^{1}$, Jeffrey L. Duerk ${ }^{2}$, Jonathan Lewin ${ }^{2}$, \\ Frank K. Wacker ${ }^{2}$, and Frank Sauer ${ }^{1}$ \\ 1 Imaging and Visualization Dept., Siemens Corporate Research, \\ 755 College Road East, Princeton NJ 08540, USA \\ $<$ first name $>$. <last name>@scr.siemens.com \\ 2 Radiology Department, Case Western Reserve University \\ 11100 Euclid Ave., Cleveland OH 44106, USA \\ <first name>.<last name>@uhrad.edu
}

\begin{abstract}
Internal organ motion due to breathing is a phenomenon that nullifies the rigidity assumptions in many interventional applications, ranging from image guided needle biopsies to external beam radiation therapy. In this paper, we propose a method to correlate and characterize internal organ motion with the location of skin markers. The method utilizes a MR time sequence along with tracked magnetic marker positions to establish the correlation. We perform a validation study to quantify the degree of the accuracy and the reproducibility of this correlation. The results demonstrate that patient specific correlation of internal motion and skin markers can be established and the target positioning accuracy of better than $15 \%$ of the maximum range of the target movement can be achieved.
\end{abstract}

\section{Introduction and Background}

Interventional procedures have been performed to localize, biopsy, and treat cancers for several decades. These procedures are gaining increased popularity mainly due to reduced trauma and improved recovery time. In minimally invasive procedures, in contrast to open procedures, there is a dramatic reduction in the surgeon's visual ability. Radiological, ultrasonic, and magnetic resonance imaging (MRI) techniques are employed to map anatomical geometry in intraoperative procedures. Image guidance systems have been devised to provide visualization of both surgical tools and preoperative images with in the same coordinate system 4. The problem arises in the scenarios, where the guidance system relies on preoperative images, assuming that the anatomical site has not deformed. The assumption is valid for certain anatomical sites (e.g., brain), where the procedures can be performed with high level of accuracy. However, many other organs, specially in thoracic and abdominal areas, are affected by respiratory and heart motion. Periodicity of the heart beat together with a reliable gating signal (i.e., ECG) provide means to address heart motion problem 
[9]. On the other hand, respiratory motion is known to be rather subjective, posing a difficult targeting problem [12. One approach is to utilize a real-time imaging modality during procedure updating the image guidance system with the most recent state of the anatomy (see e.g. 2], 44). Real-time imaging modalities, such as ultrasound and x-ray, do not provide high quality images with soft tissue contrast. Therefore, the utilization of such imaging systems is limited to certain procedures. In [1], the authors address this problem by registering a real-time low quality intraoperative set of images with preoperative high quality MR images. The proposed registration problem is then solved using a priori knowledge of organ movement within the preoperative data sets taken at various stages of breathing using the breath-hold technique. There have been also studies where researcher try to model and/or characterize the respiratory motion using realtime imaging [3.79:10 11]. The target movement characterization has also been addressed in radiotherapy applications 813. In this application, either the target motion is incorporated in 3D dose calculation and planning [8], or the radiation beam is gated using a sensor indicating the stage of breathing [8]. The effectiveness of the latter method depends on the degree of the dependency and reproducibility of breathing sensor signal to tumor location. Unfortunately, there is little information in the literature with regard to the correlation between internal anatomical and external skin marker movement.

In this paper, we propose a method to infer the internal organ motion (position) by observing the movement of a skin marker set. At first, we employ a realtime modality such as MRI in cine mode. In the pre-procedure phase, we acquire a set of images along with the position of a marker set attached on to the patient's skin surface. We analyze the markers and the target positions within the images for several breathing cycles. Once the sought after relationship is established, in the interventional stage, the skin markers are tracked and according to the position and the motion pattern the image guidance system is updated with the preoperative data, which best represent the current stage of breathing. Our goal is to 1) devise an appropriate technology for both marker and image based target tracking, 2) to design and implement an algorithm to correlate the external (artificial) and internal (natural) landmarks, 3) to evaluate the correlation of target movement and marker position, and 4) to perform patient/volunteer studies and validate the overall approach.

\section{Material and Method}

The goal is to predict the location of an anatomical target at the time of intervention without performing real-time imaging. It is assumed that the movement of the target lesion is only caused by the diaphragm motion due to breathing. A set of external markers, which are placed on the subject's thorax and can be tracked optically, provides information regarding the patient's respiratory stage during intervention. This requires a patient specific knowledge of the correlation between the skin markers and internal target movements, which has to be established prior to the intervention. The relationship depends on many individ- 
ual parameters including skin marker position, internal target location, subject weight, height, and most importantly, specific breathing pattern. In the proposed approach, we characterize the relationship shortly before the procedure. During the interventional procedure, the patient specific correlation pattern is used to infer the location of the internal targets from the movement of the skin markers.

\subsection{Pre-procedure Stage}

In the pre-procedure mode, the goal is to characterize the relationship between the movement of skin markers and internal target. This process requires acquiring a set of images through multiple breathing cycles, in which the target point can be identified and its location be tracked. Furthermore, the locations of the skin markers associated with each image have also to be acquired. We employ a MRI system to acquire both the images and to track the location of magnetic markers. The magnetic markers are tuned coils attached to a plate, which is also instrumented with a set of optical markers. The marker plate is attached to the patient's skin. The optical markers are used to track the position of the magnetic markers once the patient is outside of the MR bore. In order to establish a common coordinate frame between the magnetic and optical markers on the plate, a calibration procedure has to be performed.

Concurrent Imaging and Marker Tracking. Time series are acquired using a MRI TRUE-FISP pulse sequence, which enables the reconstruction of a 25 $\mathrm{cm}^{2}$ field of view in less than $200 \mathrm{~ms}$. The magnetic markers are LC tuned coils, where their natural frequency is matched to that of the proton resonance of the scanner. One-dimensional projection signals of a fast radial MRI pulse sequence suppress virtually all signals in the imaging volume except that from the magnetic markers. The one-dimensional projections contain peaks, which correspond to each magnetic marker. The projections are acquired in between two consecutive image acquisitions. In [5], the authors describe a method to reconstruct the three-dimensional coordinates of the markers within the imaging volume using a set of one-dimensional peaks. In our application, three magnetic markers are attached to a plate with a fixed geometry. Therefore, there is a strong geometrical constraint regarding the relative positions of the markers. We have incorporated that into the reconstruction algorithm outlined in [5]. The new approach has a non-linear optimization step. The a priori knowledge regarding the geometry of three markers, enables us to formulate an energy functional based on position and orientation (i.e., six parameters) of the coordinate frame assumed to be representing the three magnetic markers.

Let us assume the positions of the three markers are $\mathbf{n}_{i} \in \Re^{3}$ for $i \in[0,2]$ within an arbitrary coordinate frame on the marker plate. The positions of the markers in the MR coordinate system can be acquired using the following equation: $\mathbf{m}_{i}=\mathbf{R} * \mathbf{n}_{i}+\mathbf{t}$, where $\mathbf{R} \in \Re^{3 \times 3}, \mathbf{t} \in \Re^{3}$ are the rotation matrix and the translation vector. This translation and rotation maps the local coordinate frame of markers to that of the MR scanner. Given the location of the magnetic 
markers $\mathbf{m}_{i}$ within the $\mathrm{MR}$ coordinate system, the operators $\mathcal{P}_{\theta}^{X Z}$, and $\mathcal{P}_{\theta}^{Y Z}$ produce peak locations in a one dimensional projection at the angle $\theta$. The position of these peaks $p_{\theta}^{X Y}(i)$ and $p_{\theta}^{Y Z}(i)$ are functions of six parameters of pose (i.e., rotation matrix $\mathbf{R}$ and translation vector $\mathbf{t}$ ), and have to coincide with the projection peaks (i.e., $\widetilde{p}_{\theta}^{X Y}(i)$ and $\left.\widetilde{p}_{\theta}^{Y Z}(i)\right)$ acquired through the radial pulse sequence applications. The optimization cost function, which has to be minimized, can be written as follows:

$$
[\widetilde{\mathbf{R}}, \widetilde{\mathbf{t}}]=\arg \min _{[\mathbf{R}, \mathbf{t}]} \sum_{i} \sum_{\theta}\left\|\left[\widetilde{p}_{\theta}^{X Y}(i) \mid \widetilde{p}_{\theta}^{Y Z}(i)\right]-\left[p_{\theta}^{X Y}(i) \mid p_{\theta}^{Y Z}(i)\right]\right\|^{2}
$$

The optimization problem in equation (1) can be solved using the LevenbergMarquart technique. The closed-form solution outlined in [5] is considered as the initial value for the optimization process. The advantage of re-formulation of the problem to form of equation (11) is: first, the geometrical constraints of the markers are inherently included, and second, the projections in two orthogonal scan planes $X Y$ and $Y Z$ are concurrently used in the reconstruction procedure. By addition of the non-linear optimization step, the accuracy and robustness of the marker tracking and labelling is increased.

Target Tracking in MR image sequence. The next step is to track the region of interest, containing the anatomical target, in a series of MR images. The goal is to build up the trajectory of the target for multiple breathing cycles. In our approach, the user specifies a bounding box around the target area in one of the images. We apply the Laplacian of Gaussian (LoG) operator on the image, first to smooth and decrease the artifacts due to the fast acquisition, and second to enhance the edges in the images and decreases the brightness and contrast variations. A normalized cross correlation technique is then used to extract the pixel movement of the desired region in each of the two consecutive images within a series.

Correlating Internal and External Motion Through PCA analysis. Principal Component Analysis (PCA) has proven to be very useful tool for dimensionality reduction of multivariate data with many application areas in image analysis and time series prediction [6]. The typical definition of PCA calls for a given set of vectors $\mathbf{a}_{1}, \cdots, \mathbf{a}_{k}$ in an $n$-dimensional space with zero mean, arranged as the columns of an $n \times k$ matrix $A$. The output set of principal vectors are an orthonormal set of vectors representing the eigenvectors of the sample covariance matrix $\mathbf{A A}^{\top}$ associated with the $q \leq n$ largest eigenvalues. The matrix $\mathbf{U U}^{\top}$ is a projection onto the principal components space with the property that: 1) the projection of the original sample is faithful in a least-square sense. i.e., $\left.\min \sum_{i=1}^{k}\left\|\mathbf{a}_{i}-\mathbf{U}^{\top} \mathbf{a}_{i}\right\|^{2}, 2\right)$ the projection of the sample set onto the lower dimensional space maximally retains the variance, i.e., the first principal vector $\mathbf{u}_{j}$ maximizes $\sum_{i}\left|\mathbf{A}^{\top} \mathbf{u}_{j}\right|$ for $j \in[1, k]$. The representation of a 
sample point $\mathbf{a}_{i}$ in the lower dimensional feature space is defined by $\mathbf{x}_{i}=\mathbf{U}^{\top} \mathbf{a}_{i}$, 3 ) the covariance matrix $Q=\sum_{i} \mathbf{x}_{i} \mathbf{x}_{i}^{\top}$ of the reduced dimension representation is diagonal, i.e., PCA de-correlates the sample data, and 4) Euclidian distance in the feature space $\mathbf{x}_{i}$ provides an opportunity to quantify the proximity among the higher dimensional samples $\mathbf{a}_{i}$.

In our application, we analyze the correlation between skin marker positions and internal target motion using the PCA method. Let us assume that we are given the location of the three skin markers through time $\mathbf{p}_{i}^{1}, \mathbf{p}_{i}^{2}$, and $\mathbf{p}_{i}^{3}$ $\left(\mathbf{p}_{i} \in \Re^{3}\right)$, and the corresponding internal marker position $\mathbf{q}_{i} \in \Re^{3}$ ), where $i$ represents discreet time $(i \leq n)$. It is widely known that, because of the hysteresis phenomenon, the target paths through the inhalation and exhalation stages do not coincide 3,11]. Therefore, we incorporate the motion to the sample vector at each time instance $i$. For the skin markers, we construct the sample vector as follows:

$$
\mathbf{a}_{i}=\left[\mathbf{p}_{i}^{1} \mathbf{p}_{i}^{2} \mathbf{p}_{i}^{3} \Delta \mathbf{p}_{i}^{1} \Delta \mathbf{p}_{i}^{2} \Delta \mathbf{p}_{i}^{3}\right]
$$

where $\Delta$ is the consecutive position difference of the point. Based on the same principal, we build up the sample array for the target lesion as $\mathbf{b}_{i}=\left[\mathbf{q}_{i} \Delta \mathbf{q}_{i}\right]$. Principal component analysis is performed on the sample vectors with zero mean. Mean of the sample vector of the skin marker set $\overline{\mathbf{a}}$ can be used to recover the rigid patient movement and correct for the patient position.

Let us denote with $\mathbf{U}$ the matrix of principal components of the sample $\mathbf{a}_{i}-\overline{\mathbf{a}}$, and call the corresponding weights and/or features $\mathbf{w}_{i}$. The components of the target position and direction samples $\mathbf{b}_{i}-\overline{\mathbf{b}}$ are denoted by $\mathbf{Y}$, and the corresponding weights by $\mathbf{v}_{i}$. $\overline{\mathbf{b}}$ is the mean of the target sample vectors. Major modes of variations in both sample marker and target sample vectors can be identified by singling out high magnitude elements of the feature space. Furthermore, a meaningful metric distance can be used in the feature space because of the orthonormality of the feature space basis vectors. Let us consider $\widetilde{\mathbf{w}}_{i}$ and $\widetilde{\mathbf{v}}_{i}$ be the features, in which only the elements with larger magnitude is kept ( $s$ and $r$ respectively). Our goal here is then to define a relationship between primary modes of the variation represented by $\widetilde{\mathbf{w}}_{i}$ and $\widetilde{\mathbf{v}}_{i}$. The function array $\mathcal{F}$ consists of functions $\left[f_{1}, f_{2}, \cdots, f_{r}\right]$, where its elements maps $\widetilde{\mathbf{w}}_{i}$ to an element in $\widetilde{\mathbf{v}}_{i}$. Domain of the functions are skin marker feature vectors, and the range is the target feature vectors. In order to specify these functions, we first parameterize them by a set of basis functions (e.g., b-splines), and second we recover the parameters, which result in functions minimizing the least-squares error for a set of corresponding features.

\subsection{Intra-procedure Stage}

At the intra-procedure stage, the patient is moved out of the scanner bore, where is accessible for the intervention. The issue at this time is to be able to update the image guidance system with the correct location of the target lesion, corresponding to the real-time respiratory state of the patient. As mentioned in 
section 2.1, the skin marker plate includes a set of optical markers, which can be tracked with an optical tracking system. The skin marker plate's magnetic and optical marker locations are known within a single coordinate frame.

Inferring Internal Organ Position. Through optical tracking of the skin markers, we first measure the position and the velocity of the magnetic markers $\widehat{a}$. The measurement is then mapped into the feature space specified by orthonormal vectors in $\mathbf{U}$. Let us denote $\widehat{\mathbf{w}}$ as the feature vector of the mapped sample. The result of the function array $\mathcal{F}$ operation on $\widehat{\mathbf{w}}$ is the estimate of the target position in feature space $\widehat{\mathbf{v}}$. The final stage is to recover the actual value for the target position using the principal components and the feature vectors.

$$
\widehat{\mathbf{b}}=\mathbf{Y} \mathcal{F}\left(\mathbf{U}^{\top} \widehat{\mathbf{a}}\right)+\overline{\mathbf{b}}
$$

It is important to mention that in the outlined procedure, we are not assuming any periodicity for the breathing.

\section{Experimental Results}

We present results of the algorithm for multiple time series acquired from abdominal/thoracic area of two volunteers. The orientation of the MRI scan planes are selected in a way that the anatomical targets, in these cases the portal vein in the liver, moves within the plane. The resolution of the images is $128 \times 128$ with square pixels of size $1.9 \mathrm{~mm}$. The slice thickness is chosen to be $10 \mathrm{~mm}$. The scanning time is about $24 \mathrm{sec}$, providing about 100 images for each study. These studies contain about 12 to 20 breathing cycles. Along with the images, we acquired 1D projections required to track the positions of the three skin markers. Both the images and markers positions are time stamped, which provides a means to relate each marker set (interpolated) position to an image. We use the algorithm specified in section 2.1 to obtain the pose of the magnetic skin markers. The re-projection errors for the optimization are always below 0.6 pixels, which corresponds to $0.59 \mathrm{~mm}$ of mean error. The procedure for the error evaluation of the algorithm is as follows. We first establish the principal feature vectors and the mapping function using all but one time samples. We then find the estimated target location using the established correlation for the sample that was left out. To establish error statistics, we permutate this operation for all the time samples in the data set. We quantify the target registration error (TRE) in terms of millimeters for all data sets. In all the data sets, we only recognize two modes of principal variation for the marker position. These two modes cover more than $98 \%$ of the total variation. Also for the target position more than $98 \%$ of the variation falls within the first two components of the feature vectors. Therefore, the function array $\mathcal{F}$ has two functions, to be determined based on the sample points.

In Figures 1(b), a sample image with the overlaid bounding boxes around the structure is shown. Figure 1(a) shows the corresponding trajectory of the 


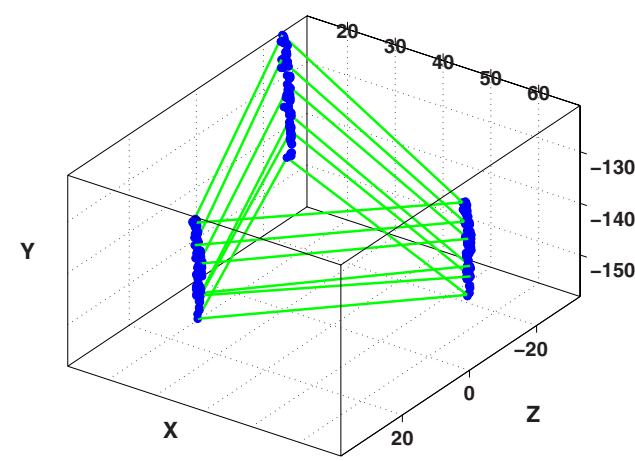

(a)

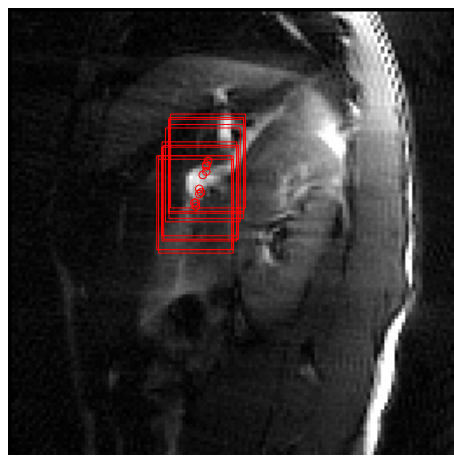

(b)

Fig. 1. (a) Trajectory of the skin marker set, and (b) MR images overlaid with the tracked target position.

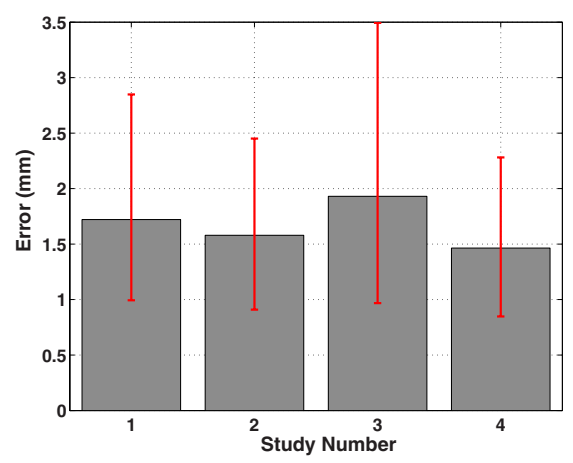

Fig. 2. Mean and standard deviation of the distance error for four different sets from two volunteers.

skin markers. Finally Figure 2, depicts the target registration error analysis for four studies performed on two volunteers, each tested twice. The mean distance error in all the studies is below $1.8 \mathrm{~mm}$. The maximum total target movement in all these data sets was about $24 \mathrm{~mm}$. Hence, for the given data set, the error in recovering the target motion with our proposed method is about $15 \%$.

\section{Summary and Conclusion}

Understanding the correlation between the movement of skin marker set and internal targets is important for many interventional applications. Based on this correlation, an image guidance system can be used in procedures on anatomical targets affected by diaphragmatic motion. In this paper, we propose a technique to characterize this correlation. The method takes advantage of a preoperative 
MRI image sequence, along with the location of tracked markers to establish this correlation. A PCA based method is used, first to identify the primary modes of variation and decrease the effects of outliers in the correlation, and second to establish a mapping function in feature domain, where a distance metric can be used. We present the results of the proposed algorithm using MR image series from two volunteers.

\section{References}

1. Blackall, J. M., King, A. P., Penney, G. P., Adam, A. and Hawkes, D. J., A Statistical Model of Respiratory Motion and Deformation of the Liver. In MICCAI 2001, Lecture Notes In Computer Science, vol. 2208, pp. 1338-1340, 2001.

2. Bzostek, A., Inoescu, G., Carrat, L., Barbe, C., Chavanon, O., Troccaz, J. Isolating Moving Anatomy in Ultrasound without Anatomical Knowledge: Application to Computer-Assisted Pericardical Punctures. In MICCAI 1998, Lecture Notes in Computer Science, vol. 1496, pp. 1041-1048, 1998.

3. Davies, S.C., Hill, A.L., Holmes, R.B., Halliwell, M., and Jackson, P.C., Ultrasound Quantitation of Respiratory Organ Motion in the Upper Abdomen. Br. J. Radiol., 67:1096-1102, 1994.

4. Desbat, L., Champleboux, G., et. al., 3D Interventional Imaging with 2D X-Ray Detectors, In MICCAI 1999, Lecture Notes in Computer Science, vol. 1679, pp. 973-980, 1999.

5. Flask, C., Elgort, D., Wong, E., Shankaranarayanan, A., Lewin, J., Wendt, M., and Duerk, J. L., A Method for Fast 3D Tracking Using Tuned Fiducial Markers and a Limited Projection Reconstruction FISP (LPR-FISP) Sequence, Journal of Magnetic Resonance Imaging, 14:617-627, 2001.

6. Jolliffe, I., T., Principal Component Analysis, Springer Verlag, New York, 1986.

7. Korin, H.W., Ehman, R.L., Riederer, S.J., Felmlee, J.P. and Grimm, R.C., Respiratory Kinematics of the Upper Abdominal Organs: A Quantitative Study. Magn. Reson. Med., 23:172-178, 1992.

8. Lujan, A.E., Larsen, E.W., Balter, J.M., and Ten Haken, R.K., A Method for Incorporating Organ Motion due to Breathing into 3D Dose Calculations. Med. Phys., 26(5):715-720, 1999.

9. McLeish, K., Hill, D.L.G., Atkinson, D., Blackall, J.M., and R. Razavi, A Study of the Motion and Deformation of the Heart due to Respiration. IEEE Trans. Med. Imaging, 21(9):1142-1150, 2002.

10. Rohling, T., Maurer, C.R., O'Dell, W.G., and Zhong, J., Modeling liver motion and deformation during the repiratory cycle using intensity-based free-form registration of gated MR images. In Medical Imaging 2001: Image Processing, 2001.

11. Suramo, I., Paivansalo, M. and Myllyla, V., Cranio-caudal Movements of the Liver, Pancreas and Kidneys in Respiration. Acta Radiologica Diagnosis, 25(2):129-131, 1984.

12. Sheafor, D.H., Paulson, E.K., Simmons, C.M., DeLong, D.M. and Nelson, R.C. Abdominal Percutaneous Interventional Procedures: Comparison of CT and US Guidance. Radiology, 207:705-710, 1998.

13. Tada, T., Minakuchi, K., et. al., Lung Cancer: Intermittent Irradiation Synchronized with Respiratory Motion - Results of a Pilot Study. Radiology , 207(3), 779-783, 1998. 